\title{
Consommation d'une association cocaïne-atropine : étude par CPG/SM et spectroscopie RMN ${ }^{1} \mathrm{H}$
}

\section{GC/MS and ${ }^{1} H$ NMR spectroscopy analyses in a case of cocaine-atropine mixture consumption}

\section{Bernard CARTIGNY(1), Michel IMBENOTTE ${ }^{(2)}$, Luc HUMBERT(1), Nicole HOUDRET ${ }^{(1)}$, Marie-Hélène TOURNOIS ${ }^{(3)}$, Nathalie AZAROUAL ${ }^{(4)}$, Gaston VERMEERSCH ${ }^{(4)}$, Michel LHERMITTE ${ }^{(1,2) *}$}

(1) Laboratoire de Biochimie et de Biologie Moléculaire, Hôpital Calmette, CHRU, 59045 Lille Cedex (2) Laboratoire de Toxicologie, Faculté des Sciences Pharmaceutiques et Biologiques, BP 83, 59006 Lille Cedex (3) Laboratoire de Biologie, Centre Hospitalier de Béthune, rue Delbecque, BP 809, 62408 Béthune Cedex (4) Laboratoire de Physique, UMR CNRS 8009, Laboratoire d'Application RMN de l'Université de Lille 2, BP 83, 59006 Lille Cedex

*Auteur à qui adresser la correspondance : Pr Michel LHERMITTE, Service de Toxicologie et Génopathies, Hôpital Calmette, 59045 LILLE Cedex - France - Tél : 33320444963 - E-mail : mlhermitte@chru-lille.fr

(Reçu le 15 octobre 2005 ; accepté le 9 mars 2006)

\section{RÉSUMÉ}

Un cas d'intoxication par un mélange cocainne atropine est rapporté chez un homme de 34 ans, retrouvé inanimé chez lui. Admis aux urgences, il présente des troubles de l'élocution et de la marche, des vertiges, une mydriase et une amnésie antérograde. L'analyse par CPG/SM de la poudre consommée a permis de mettre en évidence les deux composés majoritaires, cocaïne et atropine, ainsi que quelques traces de phénacétine. Le mélange ayant sûrement été fait succinctement, les différentes analyses quantitatives ont révélé l'hétérogénéité de composition avec en moyenne $60 \%$ de cocaïne et $30 \%$ d'atropine, confirmée par spectroscopie

\section{SUMMARY}

A 34-year-old man, poisoned by a mixture of cocaine and atropine is found inconscious, at home. He is admitted to an energengy department of a general hospital. He presents difficulties in speaking and walking, feels dizzy and also presents mydriasis. On oral examination, he does not remember anything. GC/MS analysis of the ingested powder shows the two major compounds, cocaine and atropine, as well as small amounts of phenacetine. As the mixture was.certainely done roughly, the different quantitative analyses revealed the heterogeneous composition of the powder with a mean of $60 \%$ cocaine and $30 \%$ atropine, that was later confirmed by ${ }^{\prime} H$ NMR spectroscopy. Urine and blood samples were col- 
RMN 'H. Des échantillons d'urine et de sang ont été recueillis. Le sang a été analysé par CPG/SM: la cocaüne a été quantifiée à $1557 \mu \mathrm{g} / \mathrm{L}$ et son métabolite, la benzoylecgonine, à $725 \mu \mathrm{g} / \mathrm{L}$. L'urine a été analysée par CPG/SM et spectroscopie $R M N^{\prime} H$ : par CPG/SM, la cocaïne a été quantifiée à $5,5 \mathrm{mg} / \mathrm{L}$, la benzoylecgonine à $38 \mathrm{mg} / \mathrm{L}$ et l'ecgonine méthylester (EME) à $8 \mathrm{mg} / \mathrm{L}$. Par spectroscopie RMN 'H de l'urine, un pic de résonance à 2,90 ppm a été attribué aux groupes $\mathrm{N}-\mathrm{CH}_{3}$ de la benzoylecgonine, de la cocaïne et de l'EME. Ce signal a été intégré à $180 \mu \mathrm{mol} / \mathrm{L}$, ce qui est en accord avec les résultats de CPG/SM. La spectroscopie $R M N{ }^{\prime} H$ a permis d'identifier l'atropine par un pic à 2,70 ppm dû au groupe $\mathrm{N}$ - $\mathrm{CH}_{3}$ et par des signaux centrés sur 7,40 ppm dus à ses protons aromatiques. L'intégration des signaux de l'atropine a permis de la quantifier à $700 \mu \mathrm{mol} / \mathrm{L}$. Dans l'urine, par spectroscopie $R M N{ }^{\prime} H$, des perturbations métaboliques ont pu être observées : augmentation de la taurine $(1,87$ nunol/L; $234 \mathrm{mg} / \mathrm{L})$ et de l'histidine $(0,69 \mathrm{mmnol} / \mathrm{L}$; $107 \mathrm{mg} / \mathrm{L})$, ce qui peut être mis en relation avec des effets connus de la cocaüne.

\section{MOTS-CLÉS}

Cocaïne, atropine, urine, sang, $R M N^{\prime} H, C P G / S M$.

\section{Introduction}

Si la toxicomanie à la cocaïne est assez bien caractérisée (1), ainsi que la consommation d'atropine ou de végétaux contenant cet alcaloïde (2), il n'en n'est pas de même pour l'association de ces deux composés.

La consommation de mélange est apparue dans la région Nord-Pas de Calais en décembre 2004 avant de s'étendre à d'autres régions : Tle de France, Lorraine, Franche-Comté, Midi-Pyrénées et Aquitaine. D'après l'Observatoire Français des Drogues et des Toxicomanies (3), 27 personnes (19 cas confirmés et 8 cas suspects) ont été intoxiquées par une association des deux composés, communément appelée « cristalline ». D'autres cas ont aussi été signalés aux Pays-Bas, en Italie, en Belgique, en Suisse, ainsi qu'un cas au Luxembourg.

Ces personnes signalées ont présenté le plus souvent des signes d'agitation, une mydriase ainsi qu'un état confusionnel. Parfois s'ajoutent des vomissements et des hallucinations pouvant se prolonger pendant $48 \mathrm{~h}$.

Pour mettre en évidence ces composés dans les produits consommés ainsi que dans les milieux biologiques, plusieurs protocoles analytiques peuvent être mis en ouvre. La cocaïne et ses métabolites, benzoyl ecgonine et ecgonine méthylester (EME), après extraction en phase solide (4), peuvent être quantifiés par chromatographie liquide haute performance associée à la spectrométrie de masse en tandem $(5,6)$. Une méthode de chromatographie phase gazeuse couplée à la spectrométrie de masse qui a été utilisée dans ce travail lected. Blood sample was analysed by GC/MS: cocaine was quantitated at $1557 \mu \mathrm{g} / \mathrm{L}$ and its metabolite, benzoylecgonine, at $725 \mu \mathrm{g} / \mathrm{L}$. Urine sample was analysed by GC/MS and IH NMR spectroscopy: by GC/MS, cocaine was quantitated at $5.5 \mathrm{mg} / \mathrm{L}$, benzoylecgonine at $38 \mathrm{mg} / \mathrm{L}$ and ecgonine methylester (EME) at $8 \mathrm{mg} / \mathrm{L}$. By 'H.NMR spectroscopy of urine, a resonance signal at $2.90 \mathrm{ppm}$ could be assigned to the $\mathrm{N}-\mathrm{CH}_{3}$ groups from benzoylecgonine, cocaine and EME. This peak was integrated at $180 \mathrm{\mu mol} / \mathrm{L}$; this is in good agreement with the results obtained by GC/MS. By 'H NMR spectroscopy, atropine was identified by' a resonance peak at 2.70 ppm which was assigned to its $\mathrm{N}-\mathrm{CH}_{3}$ group and by signals from aromatic protons centered at $7.40 \mathrm{ppm}$. With these signals, atropine was quantitated to $700 \mu \mathrm{mol} / L$. This method also allow to visualise some metabolic dysfunctions such as the increase of taurine (1.87. inmol/L; $234 \mathrm{mg} / \mathrm{L}$ ) and of histidine $(0.69 \mathrm{mmol} / \mathrm{L} ; 107 \mathrm{mg} / \mathrm{L})$. This can be related to known effects of cocaine.

\section{KEY-WORDS}

Cocaine, atropine, urine, blood, 'H NMR, GC/MS.

est toujours d'actualité (7).

La spectroscopie RMN ayant déjà été appliquée préalablement à l'analyse d'autres types de xénobiotiques (8).

Dans ce travail, nous avons eu recours à plusieurs techniques à l'occasion d'un cas d'intoxication par le mélange cocaïne-atropine. L'objectif de la démarche, en utilisant les techniques de CPG-SM et de spectroscopie RMN 'H, est donc de mettre en évidence les composés chimiques dans la poudre consommée et de les mesurer dans le sang et dans l'urine.

\section{Matériel et méthodes}

\section{Cas clinique}

Un homme de 34 ans, cocaïnomane depuis 13 ans, est retrouvé inconscient dans son appartement complètement en désordre, ce qui laisse supposer un état d'agitation antérieur. Il est amené aux urgences dans la nuit, environ douze heures après la prise d'une poudre d'aspect blanchâtre et cristallisé.

A l'examen, il présente une mydriase, des sensations d'engourdissement et des sensations vertigineuses. Il présente également des troubles de la marche, et de l'élocution à cause d'un trismus.

Un traitement symptomatique est mis en place et son état confusionnel s'améliore. A l'interrogatoire, il déclare «ne se souvenir de rien » et avoir été surpris par l'effet inhabituel constaté au bout de trente minutes après la prise.

Les analyses biologiques. usuelles sont réalisées et ne 
révèlent aucune anomalie si ce n'est une natrémie à 134 $\mathrm{mmol} / \mathrm{L}$. Les résultats d'hématologie et d'hémostase sont normaux. La recherche immunoenzymatique, dans le sang et les urines, de barbituriques, méthadone, opiacés, cannabinoïdes, amphétamines ainsi que l'éthanolémie se sont révélées négatives. Par contre, le dépistage immunoenzymatique de la cocaïne et des benzodiazépines est positif dans l'urine ce qui confirme en partie les données de l'interrogatoire mentionnant la prise de bromazépam $\left(\right.$ Lexomil $\left.^{\Phi}\right)$ par le patient. L'évolution est favorable et le patient sort de l'hôpital dès le lendemain.

\section{Prélèvements}

Un échantillon de sang et de l'urine ont été collectés à l'admission. Le produit supposé consommé et dénommé « cristalline » a aussi pu être analysé.

L'ensemble des prélèvements a été congelé à - $20^{\circ} \mathrm{C}$ jusqu'à l'analyse.

\section{Instrumentation et réactifs}

Les analyses de CPG/SM ont été faites sur un chromatographe en phase gazeuse Agilent 6890 couplé à un spectromètre de masse Agilent 5973N. Les composés standards deutérés ont été obtenus chez Lipomed AG (Fabrikmattenweg 4, CH-4144 Arlesheim, Suisse). Le réactif BSTFA à $1 \%$ de TMCS a été fourni par Alltech associates Inc. (2051 Wankegan road, Deerfield, IL 60015, USA).

Les analyses RMN 'H ont été réalisées sur un spectromètre Bruker AVANCE $500 \mathrm{MHz}$.

L'eau deutérée $\left(\mathrm{D}_{2} \mathrm{O}\right)$ et l'acide 3-triméthylsilyl 2,2,3,3-tétradeutéropropionique (TSP- $\mathrm{d}_{4}$ ) sont fournis par Eurisotop (St Aubin, France).

\section{Chromatographie phase gazeuse - spec- trométrie de masse}

Pour l'analyse de la cocaïne et de ses métabolites, nous avons eu recours à la méthode décrite par Gaillard et coll. (7). Un mL d'échantillon est extrait en présence des étalons internes deutérés (cocaịne- $\mathrm{D}_{3}$, benzoyl ecgonine- $\mathrm{D}_{3}$ et $E M E-\mathrm{D}_{3}$ ), en tampon phosphate $\mathrm{pH} 8,4$, par $5 \mathrm{~mL}$ d'un mélange dichlorométhane/isopropanol (9/1). Après agitation au vortex pendant $3 \mathrm{~min}$, on centrifuge $5 \mathrm{~min}$ à 4000 tours $/ \mathrm{min}$. La phase organique est récupérée et évaporée sous azote à $60^{\circ} \mathrm{C}$. L'extrait sec est dérivé par $50 \mu \mathrm{L}$ de BSTFA à $1 \%$ de TMCS pendant $15 \mathrm{~min}$ à $70^{\circ} \mathrm{C}$ et injecté en mode spitless avec une température d'injecteur de $250{ }^{\circ} \mathrm{C}$. La séparation chromatographique est réalisée sur une colonne BP X 5 MS (25 $\mathrm{m} \times 0,25 \mu \mathrm{m})$ avec le gradient de température suivant : $60^{\circ} \mathrm{C}$ pendant 2 min puis $12^{\circ} \mathrm{C} / \mathrm{min}$ jusqu'à $300^{\circ} \mathrm{C}$. L'acquisition est faite en impact électronique à $70 \mathrm{eV}$, avec une source à $150^{\circ} \mathrm{C}$, en mode full scan de 50 à $500 \mathrm{~m} / \mathrm{z}$.

\section{Spectroscopie RMN ${ }^{1} \mathrm{H}$}

Dans un tube RMN de $5 \mathrm{~mm}$ de diamètre, on introduit $500 \mu \mathrm{L}$ d'échantillon (poudre en solution, solutions de standards et urine ajustées à $\mathrm{pH} 6,0$ ) et un tube porte capillaire contenant une solution de TSP- $\mathrm{d}_{4}$ dosée à 2,69 $\mathrm{mmol}$ proton/L. Cette solution sert de référence pour les déplacements chimiques ( $\delta^{\prime} \mathrm{H}=0,00 \mathrm{ppm}$ ) et permet la quantification des composés par intégration de leurs pics relativement au TSP- $\mathrm{d}_{4}$. Une séquence de présaturation est appliquée pour atténuer le signal de l'eau. Selon la concentration des échantillons, 1.28 à 512 accumulations ont été réalisées sur une largeur spectrale de $8000 \mathrm{~Hz}$. Avant transformation de Fourier, une fonction exponentielle correspondant à un élargissement de $0,3 \mathrm{~Hz}$ est appliquée. L'utilisation du logiciel 1D WIN NMR de Bruker a servi au traitement des données.

\section{Résultats et discussion}

\section{Analyse de la poudre}

La poudre consommée a d'abord été analysée par CPG/SM. Un pic élué à 18,3 minutes présente les ions $\mathrm{m} / \mathrm{z}$ suivants : $303,272,198,182,124,105,94,82$ et correspond à celui de la cocaïne. Un autre pic majeur, élué à 18,9 minutes présente les ions $271,140,124,94,82$ et est identifié à l'atropine. Un pic mineur a aussi été détecté par CPG/SM de la poudre : il s'agit de la phénacétine éluée à 14,3 minutes et identifiable par ses ions caractéristiques $\mathrm{m} / \mathrm{z}: 179,150,137,109,108,81,80,53$.

Le spectre RMN 'H de la poudre mise en solution dans $\mathrm{D}_{2} \mathrm{O}$ révèle la présence de deux molécules majoritairement présentes : l'atropine et la cocaïne. Le spectre de l'atropine présente un singulet résonnant à $2,70 \mathrm{ppm}$ (figure 1), correspondant au groupe $\mathrm{N}-\mathrm{CH}_{3}$, le proton en alpha de la fonction ester résonnant à $5,1 \mathrm{ppm}$ (figure 2 ), et les protons aromatiques un ensemble de signaux centrés sur 7,45 ppm. Le spectre de la cocaüne présente quelques signaux caractéristiques (figures 1 et 2) en particulier deux singulets résonnant à 2,90 et 3,65 ppm et correspondant respectivement aux groupes $\mathrm{N}$ $\mathrm{CH}_{3}$ et $\mathrm{O}-\mathrm{CH}_{3}$, le proton anomérique résonnant sous forme d'un quadruplet à $5,6 \mathrm{ppm}$ et les protons du cycle aromatique monosubstitué donnant trois signaux caractéristiques à 7,55 7,70 et 8,0 ppm, facilement différenciables de ceux de l'atropine.

D'un point de vue quantitatif, les analyses, répétées sur différents aliquotes du seul échantillon de poudre disponible, en CPG/SM et en RMN 'H ont montré un rapport très variable entre la cocaïne et l'atropine. La proportion de cocaïne dans la poudre varie entre 55 et $65 \%$ du mélange et celle d'atropine entre 25 et $35 \%$. Cette hétérogénéité de composition est probablement due à la façon très succincte dont sont mélangés les produits. 
La phénacétine a pu être détectée dans la «cristalline » par RMN 'H. Seuls les deux doublets aromatiques, de très faible intensité, caractéristiques de ce composé, résonnant à 6,98 et $7,30 \mathrm{ppm}$ ont pu être mis en évidence dans la cristalline (figure 2). Les autres signaux attribuables à ce composé comportent de trop nombreuses interférences : les deux signaux attendus pour les groupements méthyle (1,36 et 2,12 ppm) sont en interférence avec des résonances observées dans les spectres de l'atropine et de la cocaïne.

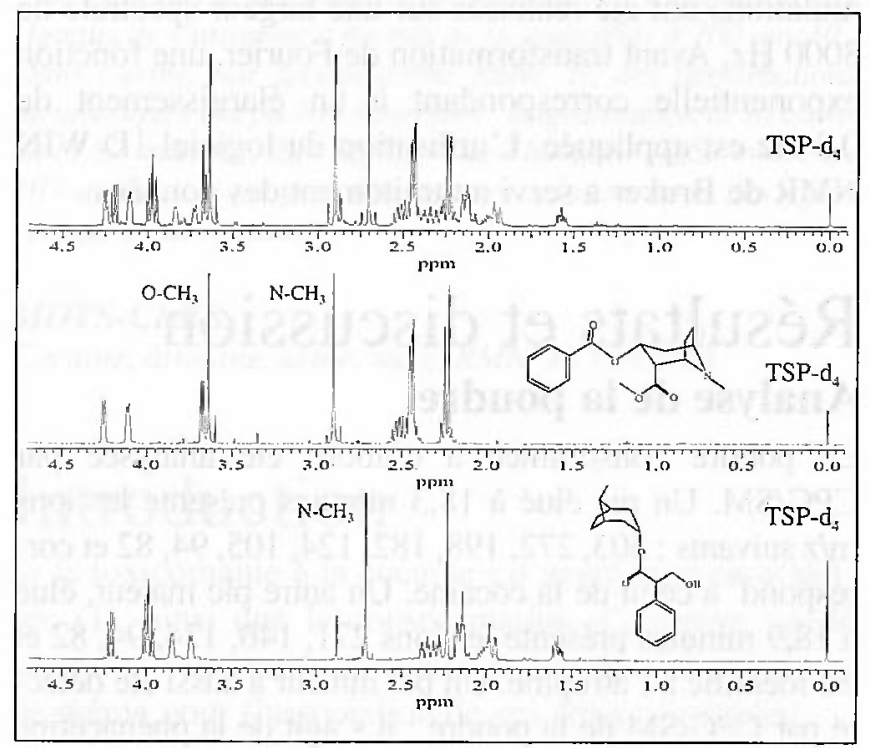

Figure 1 : Spectres $R M N^{\prime} H 500 \mathrm{MHz}$, parties aliphatiques, de la poudre consonmée, en solution dans $D_{2} O$ (en haut), d'un standard de cocaïne (au centre) et d'un standard d'atropine (en bas).

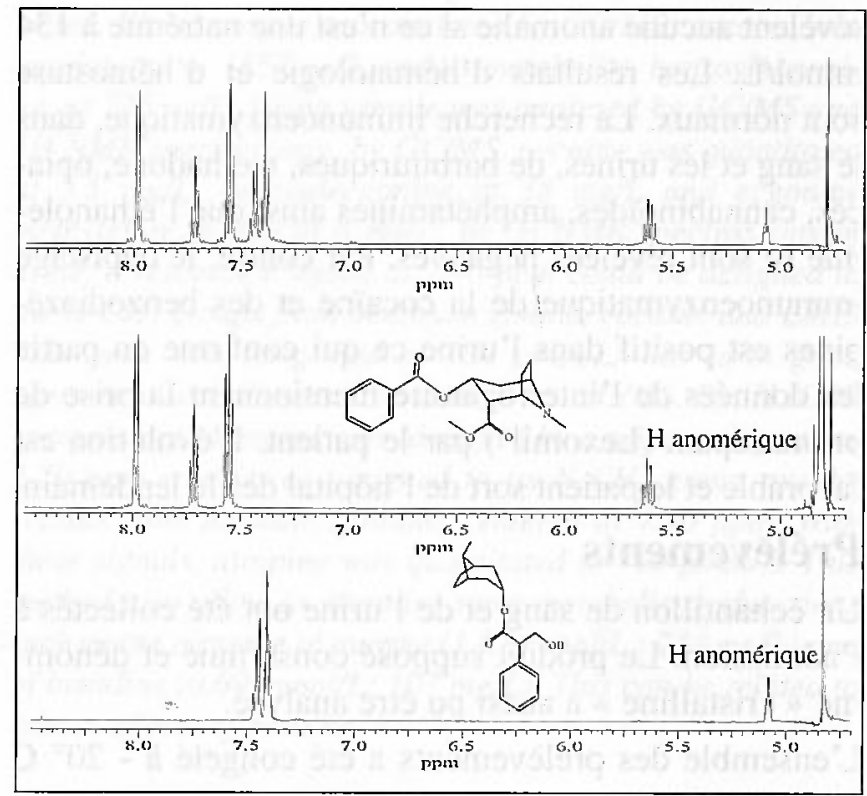

Figure 2 : Spectres RMN'H $500 \mathrm{MHz}$, parties aromatiques, de la poudre consommée, en solution dans $\mathrm{D}_{2} \mathrm{O}$ (en haut), d'un standard de cocainne (au centre) et d'un standard d'atropine (en bas).

\section{Analyse du sang}

L'échantillon de sang a été analysé uniquement par CPG/SM (figure 3) car le volume d'échantillon recueilli ne suffisait pas à réaliser l'analyse par spectroscopie RMN 'H ultérieurement. Dans les conditions analytiques, la cocaine est éluée à 18,3 minutes $(\mathrm{m} / \mathrm{z}$ caractéristique : 182) et la benzoylecgonine à 18,7 minutes $(\mathrm{m} / \mathrm{z}: 240)$. Un pic élué à 18,9 minutes et pré-

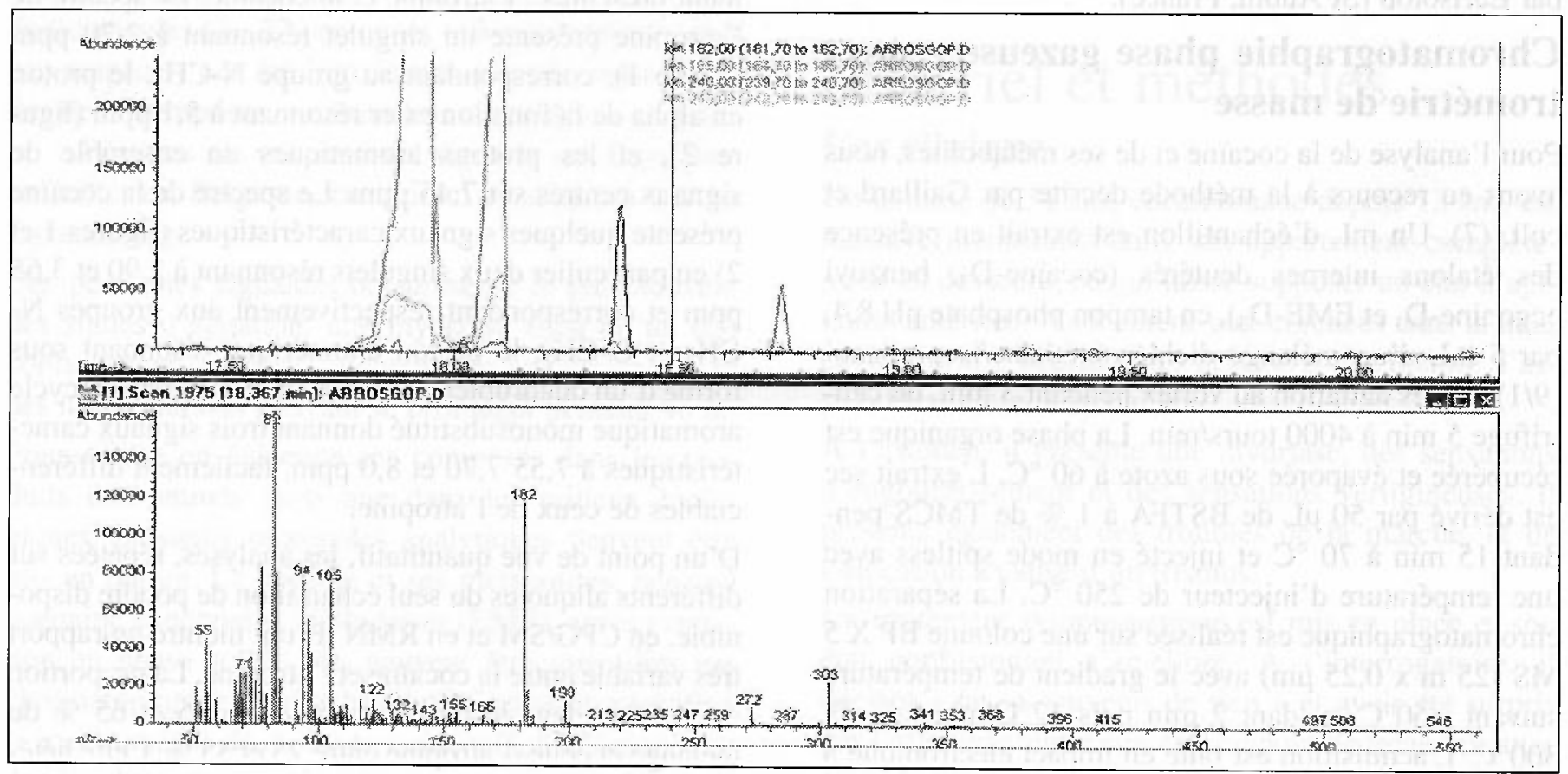

Figure 3 : Analyse du sang du patient intoxiqué par CPG-SM; cocä̈ne : 18,3 minutes et m/z:182 ; benzoylecgonine : 18,7 minutes et $\mathrm{m} / \mathrm{z}: 240$; en bas, spectre de masse d'un standard de cocaüne. 
sentant les ions caractéristiques de l'atropine a été détecté mais non quantifié. La concentration de cocaïne dans l'échantillon a été trouvée à $1557 \mu \mathrm{g} / \mathrm{L}$ de sang (Tableau I). De la benzoylecgonine a aussi été trouvée à $725 \mu \mathrm{g} / \mathrm{L}$ de sang. Ces valeurs sont relativement élevées ; Drummer et coll. (9) estiment que $500 \mu \mathrm{g}$ de cocaïne par litre de sang correspond à une prise orale de 10 à $100 \mathrm{mg}$ de cocaïne. Dans le cas présent, la quantité consommée devait être importante et a pu provoquer la saturation des enzymes de son métabolisme. Cet effet a déjà été établi pour des cholinestérases (10). L'importance de la quantité consommée peut être mise en relation avec la symptomatologie marquée au plan neurologique.

\section{Analyse de l'urine}

L'échantillon d'urine a été analysé en parallèle par deux techniques : la $\mathrm{CPG} / \mathrm{SM}$ et la spectroscopie RMN ${ }^{1} \mathrm{H}$.

L'analyse de l'urine par CPG/SM (figure 4) montre la séparation chromatographique des trois espèces chimiques : l'EME (12,7 min), la cocaïne (18,3 min) et la benzoylecgonine $(18,7 \mathrm{~min})$. La quantification de ces trois composés est présentée Tableau I ; le composé majoritaire est la benzoylecgonine.

L'analyse du spectre RMN 'H de l'urine, introduit directement dans le tube, permet la détection spécifique et la quantification de trois types de composés : les composés endogènes normaux, les xénobiotiques et les composés endogènes révélant des perturbations du métabolisme cellulaire.
Tableau I : Concentrations des xénobiotiques dans le sang et l'urine du patient intoxiqué.

\begin{tabular}{|c|c|c|c|c|}
\hline & cocaine & benzoylecgonine & $\begin{array}{c}\text { ecgonine } \\
\text { méthylester }\end{array}$ & atropine \\
\hline $\begin{array}{c}\text { sang } \\
(\mathrm{CPG} / \mathrm{SM})\end{array}$ & $1557 \mu \mathrm{g} / \mathrm{L}$ & $725 \mu \mathrm{g} / \mathrm{L}$ & - & - \\
\hline $\begin{array}{c}\text { urine } \\
(\mathrm{CPG} / \mathrm{SM})\end{array}$ & $\begin{array}{c}5,5 \mathrm{mg} / \mathrm{l} \\
18 \mu \mathrm{mol} / \mathrm{L}\end{array}$ & $\begin{array}{c}38 \mathrm{mg} / \mathrm{L} \\
133 \mu \mathrm{mol} / \mathrm{L}\end{array}$ & $\begin{array}{c}8 \mathrm{mg} / \mathrm{L} \\
40 \mu \mathrm{mol} / \mathrm{L}\end{array}$ & - \\
\hline $\begin{array}{c}\text { urine } \\
\left(\mathrm{RMN}^{\mathrm{l}} \mathrm{H}\right)\end{array}$ & \multicolumn{2}{|c|}{ somme des trois : $180 \mu \mathrm{mol} / \mathrm{L}$} & $700 \mu \mathrm{mol} / \mathrm{L}$ \\
\hline
\end{tabular}

Pour les premiers sont retrouvés (figure 5) : acides lactique (doublet du $\mathrm{CH}_{3}$ à $1,30 \mathrm{ppm}$ ), acétique (singulet à $1,90 \mathrm{ppm}$ ), alanine (doublet à $1,45 \mathrm{ppm}$ ), créatinine (singulets à 3,02 et $4,05 \mathrm{ppm}$ dus respectivement aux groupements $\mathrm{CH}_{3}$ et au $\mathrm{CH}_{2}$ ), créatine (singulets à 3,01 et 3,91 ppm), et acide hippurique (figure 6) : doublet à $7,82 \mathrm{ppm}$. La quantification de ces composés endogènes normaux est regroupée dans le tableau II.

Les xénobiotiques apparaissent sous la forme de singulets : le premier, détecté à 2,90 ppm est attribuable à la somme des groupements fonctionnels $\mathrm{N}-\mathrm{CH}_{3}$ de la cocaine, de la benzoylecgonine et de l'ecgonine méthylester (EME). L'intégration de ce signal conduit à la concentration globale pour ces trois composés de 180 $\mu \mathrm{mol} / \mathrm{L}$ (Tableau I) puisque ce signal est identique pour les trois molécules. La spectroscopie RMN'H permet aussi la quantification directe de l'atropine par l'intégration du signal à 2,70 ppm attribué au groupement fonctionnel $\mathrm{N}_{-} \mathrm{CH}_{3}$. Par cette technique, les limites de

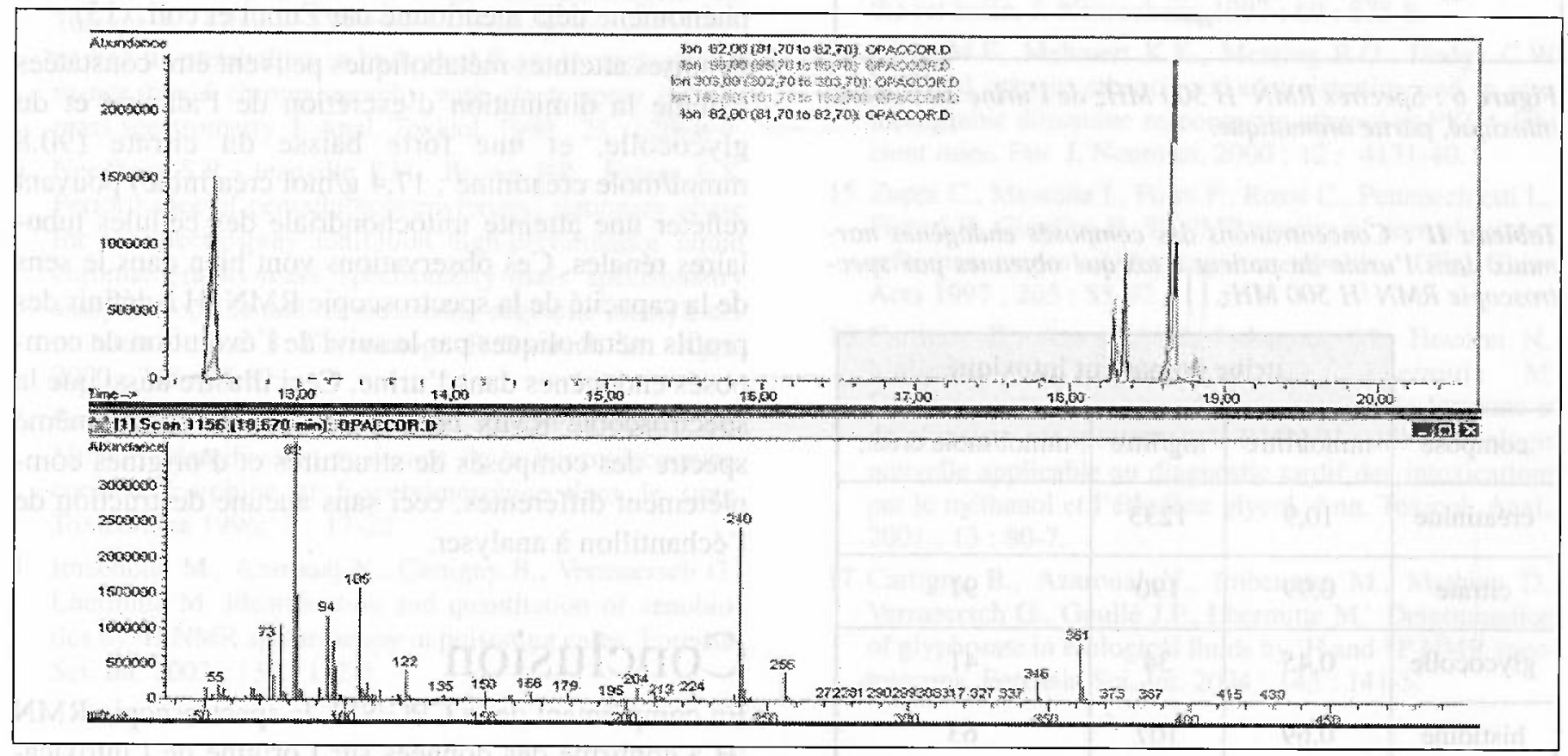

Figure 4: Analyse de l'urine du patient intoxiqué par CPG-SM; Ecgonine méthylester :12,7 minutes et m/z:82 et $96 ;$ cocaïne : 18,3 minutes et $\mathrm{m} / \mathrm{z} 182$; benzoylecgonine : 18,7 minutes et $\mathrm{m} / \mathrm{z} 240$; en bas, spectre de masse d'un standard de benzoylecgonine. 


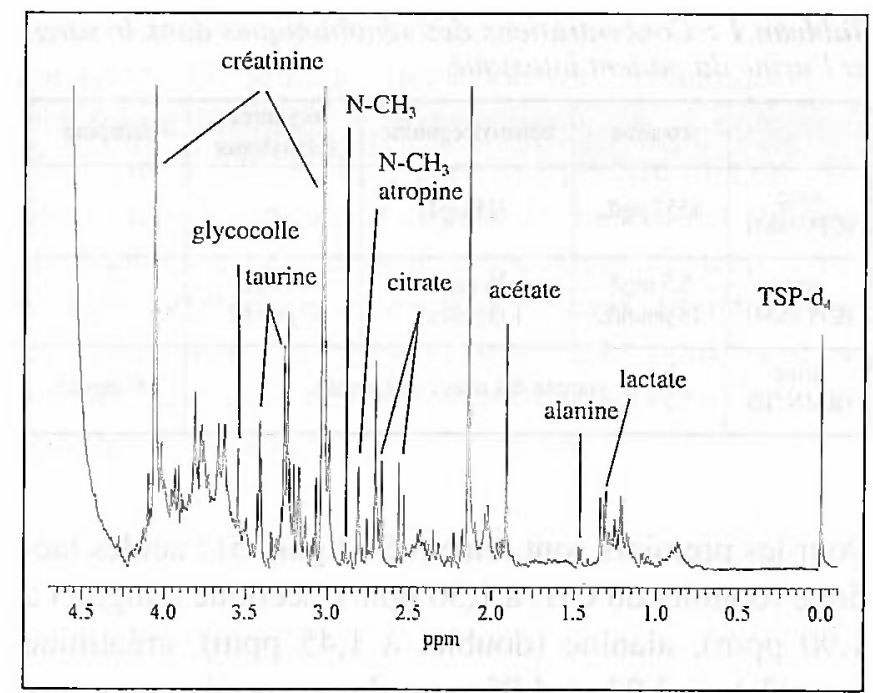

Figure 5 : Spectres $R M N^{\prime} H 500 \mathrm{MHz}$ de l'urine du patient intoxiqué, partie aliphatique.

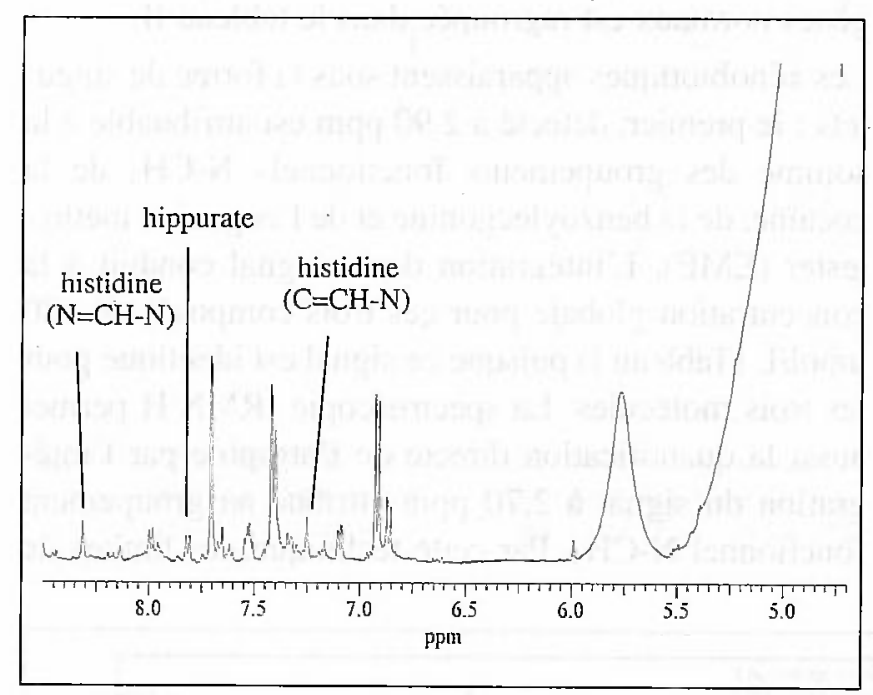

Figure 6: Spectres $R M N^{\prime} H 500 \mathrm{MHz}$ de l'urine du patient intoxiqué, partie aromatique.

Tableau II : Concentrutions des composés endogènes normaux dans l'urine du patient intoxiqué obtenues par spectroscopie RMN'H $500 \mathrm{MHz}^{\prime}$.

\begin{tabular}{|c|c|c|c|}
\cline { 2 - 4 } \multicolumn{1}{c|}{} & \multicolumn{3}{|c|}{ urine du patient intoxiqué } \\
\hline composé & $\mathrm{mmol} / \mathrm{litre}$ & $\mathrm{mg} / \mathrm{litre}$ & $\mathrm{mmol} / \mathrm{mole}$ créat. \\
\hline créatinine & 10,9 & 1233 & - \\
\hline citrate & 0,99 & 190 & 91 \\
\hline glycocolle & 0,45 & 34 & 41 \\
\hline histidine & 0,69 & 107 & 63 \\
\hline taurine & 1,87 & 234 & 172 \\
\hline
\end{tabular}

détection et de quantification de la cocaïne et de l'atropine sont respectivement de 10 et $30 \mu \mathrm{mol} / \mathrm{L}$ ( 3 et 9 $\mathrm{mg} / \mathrm{L}$ ) alors qu'en $\mathrm{CPG} / \mathrm{SM}$, elles sont de 3 et $10 \mu \mathrm{g} / \mathrm{L}$. Ceci est une limite de la spectroscopie RMN lorsque les quantités à doser sont faibles et va dans le sens d'une complémentarité entre ces deux techniques analytiques.

La concentration importante de l'atropine ( $700 \mu \mathrm{mol} / \mathrm{L}$ soit $203 \mathrm{mg} / \mathrm{L}$ ) tend à prouver le surdosage, le patient n'ayant rien précisé. La quantité de poudre consommée a du être importante et l'effet conjugué de la cocaïne et de l'atropine va bien dans le sens d'une mydriase importante (11). L'hypertonie musculaire observée au niveau des mâchoires est certainement à rapprocher du surdosage en cocaïne, peut-être potentialisé par une forte dose d'atropine.

Enfin, par RMN 'H, des perturbations métaboliques peuvent être mises en évidence (figures 5 et 6 ) : valeur élevée de l'histidine $(0,69 \mathrm{mmol} / \mathrm{L})$ et forte concentration en taurine $(1,87 \mathrm{mmol} / \mathrm{L})$. Mori et Coll. (12), et Ito et Coll. (13) avaient présenté des effets activateurs de la cocaïne sur le système histaminergique. Olive et Coll. (14) ont publié l'effet stimulant de la cocaïne sur la sécrétion de taurine chez la souris, la taurine ayant un effet potentiellement inhibiteur sur l'excitabilité neuronale. Cette augmentation significative de la taurine peut procéder de deux mécanismes:

- l'un lié à l'augmentation de production dans l'organisme, tout particulièrement au niveau du système nerveux central (14).

- l'autre traduisant un effet de réabsorption tubulaire, phénomène déjà mentionné par Zuppi et coll. (15).

D'autres atteintes métaboliques peuvent être constatées comme la diminution d'excrétion de l'alanine et du glycocolle, et une forte baisse du citrate $(90,8$ $\mathrm{mmol} / \mathrm{mole}$ créatinine ; $17,4 \mathrm{~g} / \mathrm{mol}$ créatinine) pouvant refléter une atteinte mitochondriale des cellules tubulaires rénales. Ces observations vont bien dans le sens de la capacité de la spectroscopie RMN 'H à définir des profils métaboliques par le suivi de l'évolution de composés endogènes dans l'urine. Ceci illustre aussi que la spectroscopie RMN permet de détecter sur le même spectre des composés de structures et d'origines complètement différentes, ceci sans aucune destruction de l'échantillon à analyser.

\section{Conclusion}

En complément de la CPG/SM, la spectroscopie RMN ' $\mathrm{H}$ a confirmé des données sur l'origine de l'intoxication et sur l'analyse de xénobiotiques dans l'urine. Sans extraction, il a été possible de quantifier la cocaine et 
ses métabolites, ainsi que l'atropine.

De plus, des modifications du métabolisme endogène ont pu être mises en relation avec des effets connus de la cocaïne. La forte quantité de mélange consommé pourrait expliquer des signes cliniques observés, en particulier la mydriase, bien que dans le cas de ce patient, il ait récupéré relativement rapidement par rapport aux autres cas décrits (3).

Par l'identification de signaux caractéristiques de chaque groupement fonctionnel, la spectroscopie RMN 'H permet également l'identification de composants non seulement dans les poudres consommées, mais également de les identifier en milieu biologique (16, 17), sans extraction et sans aucun a priori sur la structure des composés pouvant être présents.

\section{Remerciements}

Les auteurs tiennent à remercier tout particulièrement le Dr. Pierre-Yves Bello pour ses données présentées dans le cadre de l'Observatoire Français des Drogues et des Toxicomanies.

\section{Références}

1. Ros Soler A., Valoria Martinez A., Nieto Munuera J. Cocaine and other psychostimulant consumption: their relationship with the childhood hyperactivity syndrome. Actas Esp. Psiquiatr. 2004 ; 32 : 346-52.

2. Lohrer F., Kaiser R. Biological hallucinogens. New patterns of substance abuse in young addicts? Nervenartz. $1999 ; 70: 1029-33$.

3. Observatoire Français des Drogues et Toxicomanies. Cocaine et atropine : 27 cas d'intoxication en France, $2^{\text {iène }}$ vague de signalement en Europe. Note d'information du 21 novembre 2005.

4. Jamdar S.C., Pantuck C.B., Diaz J., Mets B. A rapid, sensitive assay for cocaine and its metabolites in biological fluids using solid-phase extraction and high-performance liquid chromatography. J. Anal. Toxicol. $2000 ; 24$ : 43841 .

5. Cailleux A., Le Bouil A., Auger B., Bonsergent G., Turcant A., Allain P. Determination of opiates and cocaine and its metabolites in biological fluids by high-performance liquid chromatography with electrospray tandem mass spectrometry. J. Anal. Toxicol. 1999 ; 23 : 620-4.

6. Needham S.R., Jeanville P.M., Brown P.R., Estape E.S. Performance of pentafluorophenylpropyl stationary phase for the electrospray ionisation high-performance liquid chromatography-mass spectrometry-mass spectrometry assay of cocaine and its metabolite ecgonine methylester in human urine. J. Chromatogr. B Biomed. Sci. Appl. $2000 ; 748: 77-87$.

7. Gaillard Y., Pépin G., Marquet P., Kintz P., Deveaux M., Mura P. Identification et dosage de la benzoylecgonine, cocaïne morphine et 6-acétylmorphine dans le sang. Toxicorama 1996; $2: 17-22$.

8. Imbenotte M., Azaroual N., Cartigny B., Vermeersch G., Lhermitte $M$. Identification and quantitation of xenobiotics by ' $\mathrm{H}$ NMR spectroscopy in poisoning cases. Forensic Sci. Int. $2003 ; 133: 132-5$.
9. Drummer O.H. Postmortem toxicology of drug of abuse. Forensic Sci. Int. 2004 ; 142 :101-3.

10. Masson P., Froment M.T., Fort S., Ribes F., Bec N., Balny C., Schopfer L.M. Butyrylcholinesterase-catalyzed hydrolysis of $\mathrm{N}$-methylindoxylacetate: analysis of volume changes upon reaction and hysteretic behavior. Biochim. Biophys. Acta 2002 ; 1597 : 229-43.

11. Schneider F., Lutun P., Kintz P., Astruc D., Flesch F., Tempé J.D. Plasma and urine concentrations of atropine after the ingestion of cooked deadly nightshade berries. Clin. Toxicol. $1996 ; 34$ : 113-7.

12. Mori T., Narita M., Onodera K., Suzuki T. Modulation of the discriminative stimulus effects of cocaine and metamphetamine by the histaminergic system. Nihon Shinkei Seishin Yakurigaku Zasshi 2002 ; 22 : 73-8.

13. Ito C., Onodera K., Sakurai E., Sato M., Watanabe T. Effect of cocaine on the histaminergic neuron system in the rat brain. J. Neurochem. $1997 ; 69: 875-8$.

14. Olive M.F., Mehmert K.K., Messing R.O., Hodge C.W. Reduced operant ethanol self-administration and in vivo mesolimbic dopamine responses to ethanol in PKC(-deficient mice. Eur. J. Neurosci. 2000; 12 : 4131-40.

15. Zuppi C., Messana I., Forni F., Rossi C., Pennacchietti L., Ferrari F., Giardina B. 'H NMR spectra of normal urines: reference ranges of the major metabolites. Clin. Chim. Acta $1997 ; 265: 85-97$.

16. Cartigny B., Azaroual N., Imbenotte M., Houdret N., Malandain $H$, Vermeersch $G$., Lhermitte $M$. Détermination de la concentration sérique du formiate et du glycolate par spectroscopie RMN 'H : une technologie nouvelle applicable au diagnostic tardif des intoxications par le méthanol et l'éthylène glycol. Ann. Toxicol. Anal., $2001 ; 13: 80-7$.

17. Cartigny B., Azaroual N., Imbenotte M., Mathieu D., Vermeersch G., Goullé J.P., Lhermitte M. Determination of glyphosate in biological fluids by ' $\mathrm{H}$ and ${ }^{31} \mathrm{P} N M R$ spectroscopy. Forensic Sci. Int. $2004 ; 143$ : 141-5. 